\title{
Effectiveness and safety of standardised shorter regimens for multidrug-resistant tuberculosis: individual patient data and aggregate data meta-analyses
}

\author{
Faiz Ahmad Khan ${ }^{1,2}$, M.A. Hamid Salim³ ${ }^{3}$ Philipp du Cros $^{4}$, Esther C. Casas ${ }^{5,6}$, \\ Atajan Khamraev ${ }^{7}$, Welile Sikhondze ${ }^{8}$, Andrea Benedetti ${ }^{1,2,9}$, Mayara Bastos ${ }^{1,10}$, \\ Zhiyi Lan ${ }^{1,9}$, Ernesto Jaramillo ${ }^{11}$, Dennis Falzon ${ }^{11}$ and Dick Menzies ${ }^{1,2,9}$
}

Affiliations: ${ }^{1}$ Respiratory Epidemiology and Clinical Research Unit, Centre for Outcomes Research and Evaluation, Research Institute of the McGill University Health Centre, Montreal, QC, Canada. ${ }^{2}$ McGill International TB Centre, McGill University, Montreal, QC, Canada. ${ }^{3}$ MDR-TB TA Project, The National TB Programme of Bangladesh, Dhaka, Bangladesh. ${ }^{4}$ Manson Unit, Médecins Sans Frontières, London, UK. ${ }^{5}$ Médecins Sans Frontières, Amsterdam, The Netherlands. ${ }^{6}$ Médecins Sans Frontières, Manzini, Swaziland. ${ }^{7}$ Ministry of Health, Tashkent Medical Pediatric Institute, Nukus, Uzbekistan. ${ }^{8}$ National TB Control Programme, Ministry of Health, Manzini, Swaziland. ${ }^{9}$ Depts of Medicine and Epidemiology, Biostatistics and Occupational Health, McGill University, Montreal, QC, Canada. ${ }^{10}$ Social Medicine Institute, State University of Rio de Janeiro, Rio de Janeiro, Brazil. ${ }^{11}$ Global TB Programme, World Health Organization, Geneva, Switzerland.

Correspondence: Faiz Ahmad Khan, Office D3.60, McGill University Health Centre, 5252 Boulevard de Maisonneuve, Montreal, QC H4A 3S5, Canada. E-mail: faiz.ahmadkhanamcgill.ca

@ERSpublications

Shorter TB regimens were effective, but generalisability of success rates to less selected populations is uncertain http://ow.ly/ptI630b3xxT

Cite this article as: Ahmad Khan F, Salim MAH, du Cros P, et al. Effectiveness and safety of standardised shorter regimens for multidrug-resistant tuberculosis: individual patient data and aggregate data metaanalyses. Eur Respir J 2017; 50: 1700061 [https://doi.org/10.1183/13993003.00061-2017].

ABSTRACT We assessed the effectiveness and safety of standardised, shorter multidrug-resistant tuberculosis (MDR-TB) regimens by pooling data from observational studies.

Published studies were identified from medical databases; unpublished studies were identified from expert consultation. We conducted aggregate data meta-analyses to estimate pooled proportions of treatment outcomes and individual patient data (IPD) meta-regression to identify risk factors for unsuccessful treatment in patients treated with 9- to 12-month MDR-TB regimens composed of a secondline injectable, gatifloxacin/moxifloxacin, prothionamide, clofazimine, isoniazid, pyrazinamide and ethambutol.

We included five studies in which 796 out of 1279 (62.2\%) individuals with confirmed MDR-TB (98.4\%) or rifampin-resistant TB (1.6\%), and not previously exposed to second-line drugs, were eligible for shorter regimens. 669 out of 796 participants were successfully treated (83.0\%, 95\% CI 71.9-90.3\%). In IPD meta-regression (three studies, $\mathrm{n}=497$ ), failure/relapse was associated with fluoroquinolone resistance (crude OR 46, 95\% CI 8-273), pyrazinamide resistance (OR 8, 95\% CI 2-38) and no culture conversion by month 2 of treatment (OR 7, 95\% CI 3-202). Two participants acquired extensive drug resistance. Four studies reported grade 3 or 4 adverse events in 55 out of 304 (18.1\%) participants.

Shorter regimens were effective in treating MDR-TB; however, there is uncertainty surrounding the generalisability of the high rate of treatment success to less selected populations, to programmatic settings and in the absence of drug susceptibility tests to key component drugs. 


\section{Introduction}

Each year, 480000 people develop active tuberculosis (TB) with strains resistant to at least isoniazid and rifampin, termed multidrug-resistant (MDR)-TB [1]. The 2011 World Health Organization (WHO) recommendations on the composition and duration of MDR-TB treatment regimens were primarily informed by an individual patient data (IPD) meta-analysis of 9153 patients that showed successful treatment was more likely if a second-line injectable was used for $\sim 8$ months (the "intensive phase") and if the total treatment duration was $\geqslant 20$ months in previously untreated MDR-TB patients $[2,3]$. Because of the burden of prolonged therapy on patients and TB programmes, and also because second-line drugs are expensive, poorly tolerated and associated with high rates of adverse events $[4,5]$, there is substantial interest in developing shorter MDR-TB regimens.

In 2010, VAn Deun et al. [6] reported an observational study, undertaken in Bangladesh by the Damien Foundation and the International Union Against Tuberculosis and Lung Disease (the Union), in which sequential cohorts of MDR-TB patients were treated with standardised regimens. The most effective regimen (now known as "the Bangladesh regimen") was 9-11 months in duration, used kanamycin, high-dose gatifloxacin, prothionamide, high-dose isoniazid, clofazimine, pyrazinamide and ethambutol, and achieved recurrence-free cure in $88 \%$ of patients $[6,7]$.

We report here a meta-analysis of observational studies of regimens of $\leqslant 12$ months in duration whose composition was based on the Bangladesh regimen, i.e. standardised shorter regimens. Our primary objective was to estimate the probability of treatment success in MDR-TB patients treated with these regimens. Secondary objectives were to identify risk factors for failure/relapse, death and loss to follow-up, to estimate risks of acquired drug resistance, and to calculate the proportion of participants experiencing adverse events. Our results were used to inform the 2016 update of the WHO MDR-TB treatment guidelines [8].

\section{Methods}

\section{Design}

We used aggregate and IPD meta-analyses of observational studies identified through systematic review and expert consultation.

\section{Study selection}

Studies reporting MDR-TB treatment outcomes, published from 2009 to August 2015, were identified by searching Medline, Embase and the Cochrane Library. Search terms, detailed in the supplementary material, included "multidrug-resistant tuberculosis", drug names and treatment outcomes. Two reviewers (Z.L. and M.B.) selected titles and abstracts for full-text review, separating studies that used regimens of conventional duration ( $\geqslant 18$ months) from those that used shorter regimens. Unpublished studies of shorter regimens were identified by a panel of experts from the WHO and the Union.

The shorter regimen studies identified by the literature search, and by the panel, were further assessed for inclusion in the current meta-analysis (a meta-analysis of conventional duration studies is reported elsewhere [9]). Inclusion criteria were: prospective design; participants with MDR-TB or rifampin-resistant TB confirmed either by culture- or nucleic acid amplification-based drug susceptibility tests (DSTs); treatment with shorter standardised regimens, of up to 12 months in duration, of composition based on the Bangladesh regimen; approval by a research ethics board and informed consent of participants (given international recommendations that shorter regimens only be used in the context of research); standardised measurement of TB treatment outcomes; and availability of the following information on the majority of participants: age at treatment initiation, sex, history of prior TB treatment, DST methods and

This article has supplementary material available from erj.ersjournals.com

Received: Jan 112017 | Accepted after revision: April 152017

Disclaimer: D. Falzon and E. Jaramillo are staff members of the World Health Organization (WHO). They alone are responsible for the views expressed in this publication and they do not necessarily represent the decisions or policies of WHO. The designations used and the presentation of the material in this publication do not imply the expression of any opinion whatsoever on the part of the WHO concerning the legal status of any country, territory, city or area, or of its authorities, nor concerning the delimitation of its frontiers or boundaries.

Support statement: This work was supported by the US Agency for International Development (including TREAT-TB which funded M.A.H. Salim's participation), World Health Organization and Fonds de Recherche du Québec - Santé (salary support to F. Ahmad Khan and A. Benedetti). Funding information for this article has been deposited with the Crossref Funder Registry.

Conflict of interest: Disclosures can be found alongside this article at erj.ersjournals.com 
results, and details on the regimen utilised (combination and dose of drugs). We excluded studies of shorter regimens that were individualised, or not based on the Bangladesh regimen, or that did not meet the inclusion criteria enumerated above.

\section{Data extraction}

For all studies, we collected inclusion and exclusion criteria (supplementary table S1), the number of patients with confirmed MDR-TB that were eligible for initiation of MDR-TB treatment during the study period, and the number initiating shorter MDR-TB regimens. For studies where only aggregate results (not IPD) were available, one of the investigators (F.A.K.) used a standard form to extract data on the following key variables: treatment regimens, outcomes (including adverse events), age, sex, history of prior TB treatment, HIV status and antiretroviral usage, results of sputum acid-fast bacilli smear microscopy, chest radiographs, and DSTs (molecular and phenotypic). For studies that provided IPD, the key variables were summarised directly from the data. Authors were contacted to provide information on participant selection and key variables that were not available in the publications.

\section{Exclusion of individual participants}

As isoniazid is used in the shorter regimen, we excluded participants with isoniazid-susceptible strains in order to avoid overestimating efficacy in patients with confirmed MDR-TB (this applied to a small number of patients, see table 1 footnote for details); however, participants with documented rifampin-resistant strains in whom DSTs for isoniazid had not been performed were included. From ongoing studies, we excluded participants whose treatment had started within 1 year of the date that data were provided to us.

\section{Outcome definitions}

We collected data on the following outcomes: success (cure or treatment completion), failure, death, loss to follow-up and relapse, keeping the original definitions applied in each study (enumerated in supplementary table S2). As relapse was rare, we combined failure and relapse into a single outcome. The month of culture conversion was that of the first of two consecutive negative cultures, performed at least 30 days apart. Adverse events were stratified by severity using the National Institute of Allergy and Infectious Diseases, Division of AIDS (DAIDS) system (rsc.tech-res.com).

\section{Statistical analyses}

We used the exact binomial likelihood method for all meta-analyses, which is less likely to produce biased estimates of heterogeneity for binary outcome data [10]. Random-effects models were used whenever possible; fixed-effects models were used when random-effects models did not converge (which happened due to small sample sizes or few outcome events). In random-effects meta-analyses, between-study heterogeneity was quantified by the variance of the random-effects parameter [10] and considered significant when this variable's confidence interval did not overlap with 0 .

We first calculated study-level outcomes for each study that had provided IPD and then pooled these with aggregate data from the remaining studies. The pooled proportion of patients successfully treated was estimated using three definitions of unsuccessful treatment outcome: 1) failure/relapse, 2) failure/relapse or death and 3) failure/relapse, death or loss to follow-up.

We then proceeded to IPD meta-regression in order to determine if baseline characteristics or timing of culture conversion were associated with 1) failure/relapse versus treatment success, 2) death versus survival (either treatment failure/relapse or success) and 3) loss to follow-up versus survival. For these analyses, we used generalised linear mixed models estimated using adaptive Gaussian hermite quadrature. Covariates assessed were: demographic variables, HIV infection, measures of disease severity (smear positivity, cavities on chest radiograph), timing of culture conversion and baseline drug resistance. Effect measures were reported as odds ratios with $95 \%$ confidence intervals. Analyses were univariable given the small number of events and studies.

Acquired drug resistance was infrequently ascertained; hence, we reported only the numbers tested and found to have acquired drug resistance.

For each type of adverse event, we calculated the proportion of participants that were affected within each study, stratifying by severity when possible. We also calculated the pooled proportion of participants experiencing any type of grade 3 or 4 adverse event (i.e. pooling all types of events).

All analyses were performed using SAS version 9.4 (SAS Institute, Cary, NC, USA). Forest plots were generated with R version 3.2.4 (R Foundation for Statistical Computing, Vienna, Austria). 
TABLE 1 Description of studies: data available, exclusion criteria, treatment characteristics and enrolment

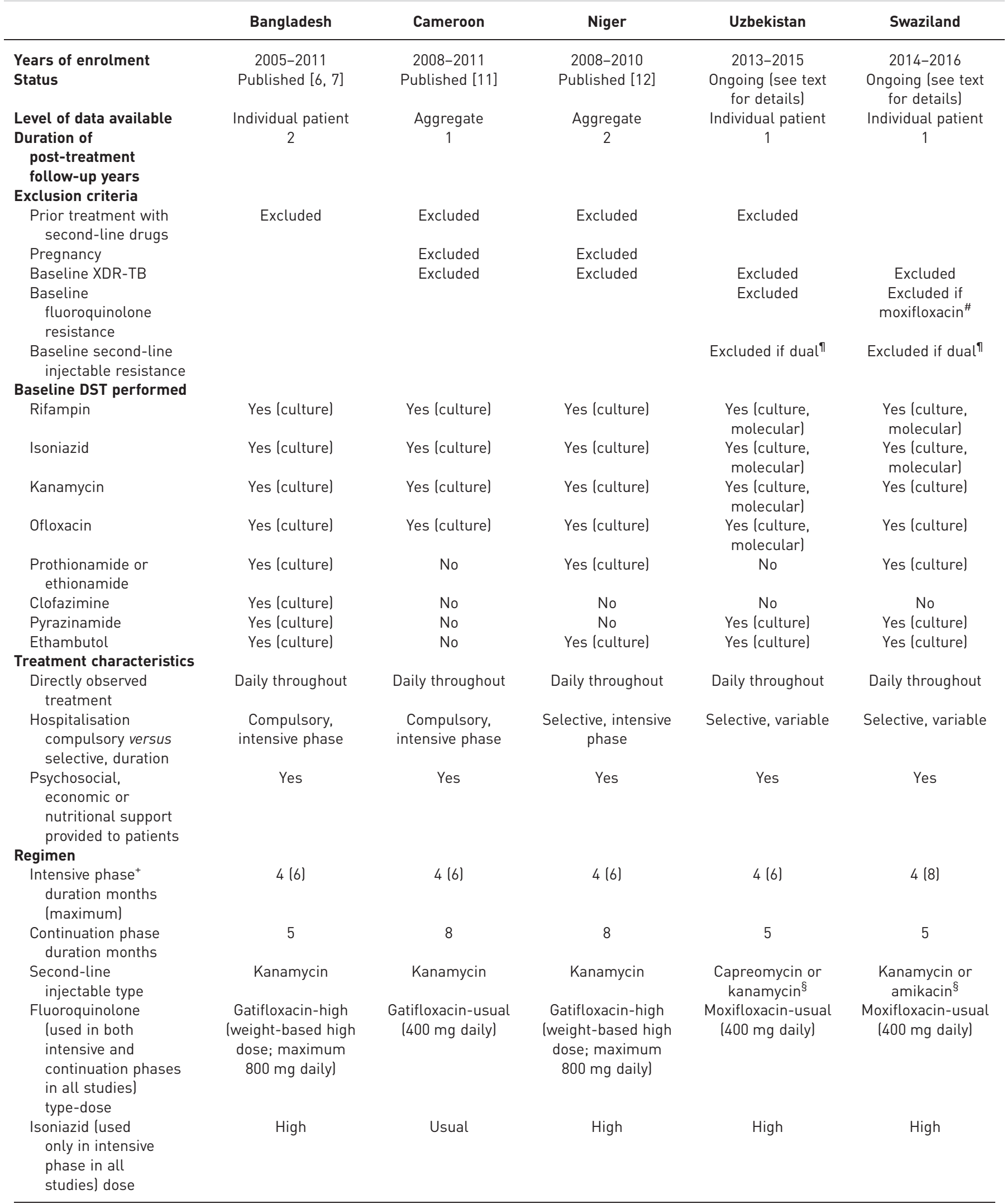




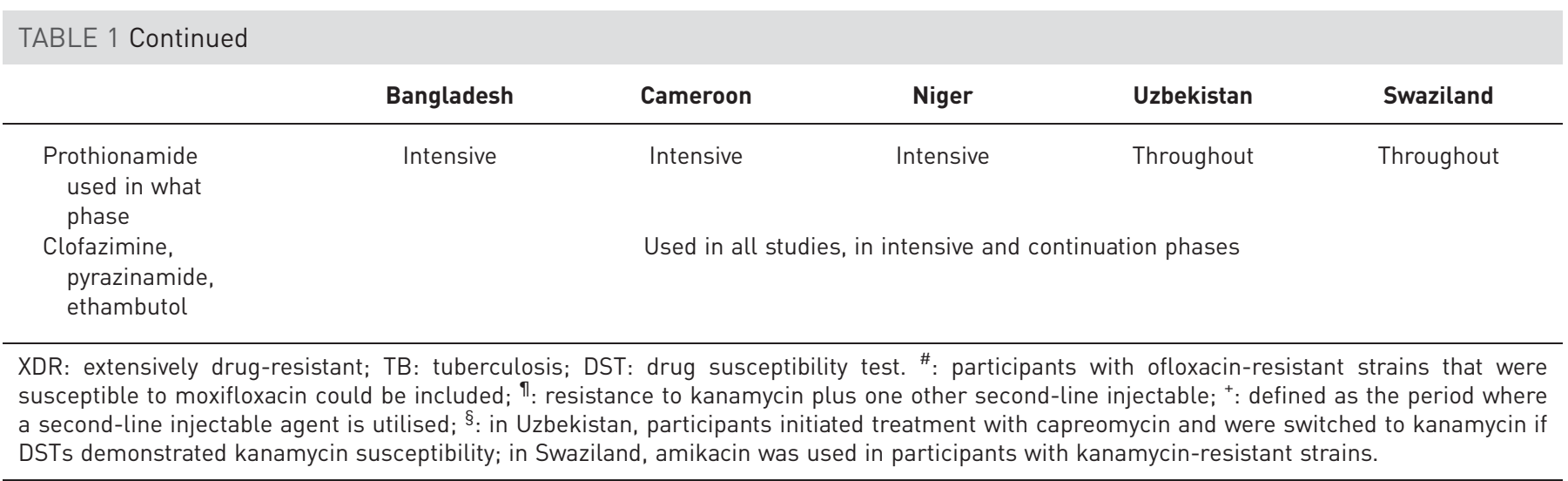

\section{Results}

Study selection is depicted in supplementary figure S1. Three published observational studies (reported in four papers $[6,7,11,12]$ ) of shorter standardised regimens were identified in the search of medical databases and all were included. Three unpublished studies were identified by the expert panel; one was excluded because interim data were not shared. Hence, a total of five studies were included (table 1): three published (Bangladesh [6, 7], Cameroon [11] and Niger [12]) and two unpublished (Uzbekistan (Médecins Sans Frontières Operational Center Amsterdam (MSF-OCA), London, UK/Ministry of Health, Nukus, Uzbekistan) and Swaziland (MSF-OCA, Amsterdam, The Netherlands/Ministry of Health, Manzini, Swaziland)). One published study (Bangladesh) and both unpublished studies (Uzbekistan and Swaziland) provided IPD. Relapse data were available in all studies for at least 1 year of post-treatment follow-up.

\section{Description of studies}

Key study-level characteristics are summarised in table 1. Prior treatment with second-line TB drugs ( $>30$ days of use) was an exclusion criteria in four studies (Bangladesh, Cameroon, Niger and Uzbekistan), as was baseline extensively drug-resistant (XDR)-TB (Cameroon, Niger, Uzbekistan and Swaziland). Ofloxacin resistance was an exclusion criterion in the Uzbekistan study and moxifloxacin resistance was an exclusion criterion in the Swaziland study. Fluoroquinolone resistance was not an exclusion criterion in the three published studies (Bangladesh, Cameroon and Niger). Comorbidities applied as exclusion criteria included critical condition/severe illness (Cameroon and Swaziland), cardio-respiratory insufficiency (Bangladesh), severe liver disease (Bangladesh and Niger), severe renal impairment (Uzbekistan and Swaziland), diabetes (Niger) and confirmed corrected QT prolongation (Uzbekistan and Swaziland) (see supplementary table S1 for additional details).

In all studies, treatment was provided under daily direct observation throughout both phases and participants also received psychosocial, financial or nutritional support. Hospitalisation during the intensive phase was compulsory in Bangladesh and Cameroon, and was used selectively at other sites.

As shown in table 1, the duration of the intensive and continuation phases, as well the composition of the regimen, varied between the studies. The intensive phase could be extended up to 8 months in the Swaziland study. The continuation phase duration was 5 months in three studies (Bangladesh, Uzbekistan and Swaziland) and 8 months in two studies (Niger and Cameroon). Gatifloxacin was used in three studies (Bangladesh, Cameroon and Niger), of which two used it at high doses (Bangladesh and Niger). Usual-dose moxifloxacin was used instead of gatifloxacin in Uzbekistan and Swaziland.

\section{Participant selection}

Among patients with confirmed MDR-TB or rifampin-resistant TB, 373 out of 1279 (29.2\%) did not initiate treatment with a shorter regimen and were excluded from our study. Reasons why participants with confirmed MDR-TB did not initiate a shorter regimen are enumerated in table 2; the most common reasons were prior exposure to second-line drugs $(n=85)$, pre-treatment loss to follow-up $(n=74)$, baseline drug resistance pattern $(n=42$, of whom three had XDR-TB) and pre-treatment death $(n=29)$.

We also excluded 110 out of $1246(8.8 \%)$ patients that initiated a shorter regimen; 86 out of 110 were excluded because their treatment started within 1 year of provision of data to our study and 15 out of 110 were excluded due to baseline drug resistance (either XDR-TB, combined capreomycin and kanamycin or 


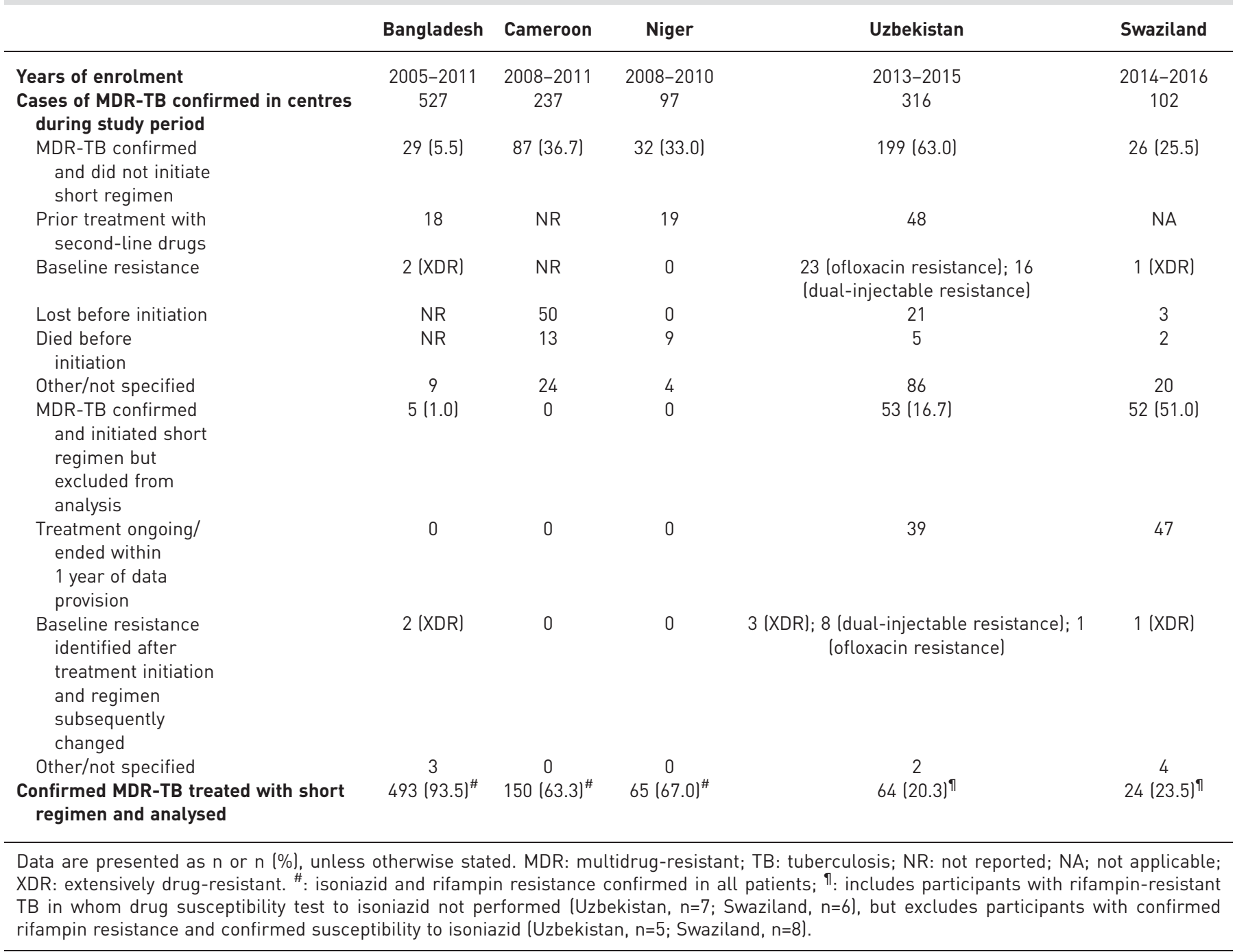

ofloxacin). Among included participants, 782 out of 796 (98.4\%) had DST confirmation of rifampin and isoniazid resistance, and 13 out of $796(1.6 \%)$ had confirmation of rifampin resistance but isoniazid susceptibility tests had not been performed.

\section{Baseline characteristics}

Table 3 summarises the baseline characteristics. Study populations had similar age distributions, centred around the fourth decade. Variability was seen in the proportion of female participants (lowest in Bangladesh and Niger) and in primary MDR-TB (common in the Uzbekistan and Swaziland studies, and rare in the others). The greatest proportion of people living with HIV was in the Swaziland cohort, followed by Niger. The majority of people living with HIV initiated antiretroviral treatment either before or during MDR-TB treatment (Swaziland: 15 out of 16; Cameroon: 22 out of 30).

Fluoroquinolone resistance was most common in the Bangladesh cohort (13\%), followed by the Swaziland cohort (7\%). Baseline resistance to second-line injectable agents was observed only in the Uzbekistan cohort among $31 \%$ of included participants. Pyrazinamide resistance was seen in the majority of participants in Uzbekistan and Swaziland, and unmeasured in Niger and Cameroon.

\section{Study outcomes}

Individual study and pooled treatment outcomes are reported in figure 1 . There were 17 cases of treatment failure, 51 participants died and 54 were lost to follow-up on treatment. Details on relapse are provided in supplementary table S3; there were five cases of relapse (three in Bangladesh, one in Uzbekistan and one 


\begin{tabular}{|c|c|c|c|c|c|}
\hline & Bangladesh & Cameroon & Niger & Uzbekistan & Swaziland \\
\hline Years of enrolment & $2005-2011$ & $2008-2011$ & $2008-2010$ & 2013-2015 & $2014-2016$ \\
\hline $\begin{array}{l}\text { Participants included in } \\
\text { meta-analyses }\end{array}$ & 493 & 150 & 65 & 64 & 24 \\
\hline Age years & $33.6 \pm 12.9$ & 35.1 & 31 (27-38) & $33.7 \pm 14.4$ & $35.2 \pm 14.4$ \\
\hline Female & $150 / 493(30.4)$ & 73/150 (48.7) & $12 / 65(18.5)$ & $35 / 64(54.7)$ & $13 / 24(54.2)$ \\
\hline Primary MDR & 4/493 (0.8) & $1 / 150(0.1)$ & $1 / 65(1.5)$ & $46 / 60(76.7)$ & $20 / 24$ (83.3) \\
\hline HIV-positive & $N R$ & $30 / 150(20.0)$ & $1 / 58(1.7)$ & $0 / 44$ & $16 / 24(66.7)$ \\
\hline Smear-positive & 475/493 (96.3) & $150 / 150(100)$ & $54 / 65(83.1)$ & $28 / 61(45.9)$ & $12 / 23(52.2)$ \\
\hline $\begin{array}{l}\text { Chest radiograph cavities } \\
\text { Baseline resistance }\end{array}$ & 99/493 (20.1) & NR & $23 / 65(35.4)$ & $26 / 60(43.3)$ & NR \\
\hline Fluoroquinolones $\#$ & $65 / 485(13.4)$ & $0 / 133$ & $1 / 65(1.5)$ & $0 / 50$ & $1 / 15(6.7)$ \\
\hline Kanamycin & $0 / 485$ & $0 / 135$ & $0 / 65$ & $13 / 49(26.5)$ & $0 / 14$ \\
\hline Capreomycin & NR & NR & NR & $2 / 48(4.2)$ & NR \\
\hline Prothionamide/ethionamide & $82 / 466(17.6)$ & NR & $7 / 65(10.8)$ & NR & $5 / 14(35.7)$ \\
\hline Pyrazinamide & $99 / 240(41.3)$ & NR & NR & $32 / 38(84.2)$ & $10 / 14(71.4)$ \\
\hline Ethambutol & $321 / 493(65.1)$ & NR & $45 / 65(69.2)$ & $30 / 43(69.8)$ & $12 / 17(70.6)$ \\
\hline
\end{tabular}

Data are presented as $n$, mean $\pm S D$, group mean, median (interquartile range) or $n / n(\%)$, unless otherwise stated. Denominators less than the total participants are due to missing data. MDR: multidrug-resistant; NR: not reported; DST: drug susceptibility test; MIC: minimum inhibitory concentration; TB: tuberculosis. \#: in all studies, DSTs for ofloxacin were performed to identify resistance to fluoroquinolones. In Bangladesh, 163 participants also had DSTs for gatifloxacin performed. Gatifloxacin resistance was defined as low level if MIC $0.5-1 \mathrm{mg} \cdot \mathrm{L}^{-1}$ and high level if $\mathrm{MIC} \geqslant 2 \mathrm{mg} \cdot \mathrm{L}^{-1}$ [7]. Of the 65 participants with fluoroquinolone-resistant TB from Bangladesh, the breakdown of the resistance profile was as follows: $n=13$ ofloxacin-susceptible with low-level gatifloxacin resistance, $n=20$ ofloxacin-resistant with low-level gatifloxacin resistance, $n=27$ ofloxacin-resistant with high-level gatifloxacin resistance, and $n=5$ ofloxacin-resistant and gatifloxacin DSTs not performed. We classified five participants from Bangladesh with ofloxacin-resistant and gatifloxacin-susceptible MDR-TB as fluoroquinolone-susceptible.

in Swaziland). The rate of treatment success versus failure/relapse was 97.0\% (95\% CI 80.1-99.6\%), the rate of treatment success versus failure/relapse or death was $88.1 \%$ (95\% CI 78.1-93.9\%) and the rate of treatment success versus failure/relapse, death or loss to follow-up was 83.0\% (95\% CI 71.9-90.3\%). Between-study heterogeneity was significant for success versus failure/relapse.

\section{IPD meta-regression}

Table 4 summarises associations between baseline participant characteristics and failure/relapse versus treatment success, death versus survival (excluding loss to follow-up) and loss to follow-up versus survival (excluding death). Heterogeneity estimates for failure/relapse are provided in supplementary table S4; for death and loss to follow-up, all analyses used fixed-effects models due to nonconvergence when including a random effect. No culture conversion by month 2 of treatment, use of moxifloxacin rather than gatifloxacin, fluoroquinolone resistance and pyrazinamide resistance were significantly associated with greater odds of failure/relapse. Death was associated with HIV infection. Loss to follow-up was more likely among participants that had not experienced culture conversion by month 2 of treatment.

\section{Impact of combined fluoroquinolone and pyrazinamide resistance}

As shown in table 5, the probability of successful treatment was highest among participants with MDR-TB susceptible to both fluoroquinolones and pyrazinamide, and lowest with resistance to both.

\section{Acquired drug resistance}

Limited data on acquired drug resistance, to five of the component drugs, were available from the three studies that provided IPD (Bangladesh, Uzbekistan and Swaziland) (table 6). Acquired resistance was observed in four out of 21 participants who failed or relapsed; in three, baseline DSTs had demonstrated resistance to fluoroquinolones and ethambutol, two of whom also had baseline pyrazinamide resistance. XDR-TB was acquired by two participants: one had a fluoroquinolone-resistant strain at baseline that acquired resistance to kanamycin, and the other had a strain that was susceptible at baseline to both fluoroquinolones and second-line injectables (without DST results for other medications). 
a)

$\begin{array}{lccc}\text { Country } & \text { Success } & \text { Total } & \text { Success \% (95\% Cl) } \\ \text { Bangladesh } & 415 & 424 & 97.9(96.0-99.0) \\ \text { Cameroon } & 134 & 135 & 99.3(95.9-100) \\ \text { Niger } & 58 & 58 & 100(93.8-100) \\ \text { Uzbekistan } & 45 & 54 & 83.3(70.7-92.1) \\ \text { Swaziland } & 17 & 20 & 85.0(62.1-96.8) \\ \text { Pooled } & 669 & 691 & 97.0(80.1-99.6)\end{array}$

b)

$\begin{array}{lccc}\text { Country } & \text { Success } & \text { Total } & \text { Success \% (95\% Cl) } \\ \text { Bangladesh } & 415 & 453 & 91.6(88.7-94.0) \\ \text { Cameroon } & 134 & 145 & 92.4(86.8-96.2) \\ \text { Niger } & 58 & 64 & 90.6(80.7-96.5) \\ \text { Uzbekistan } & 45 & 56 & 80.4(67.6-89.8) \\ \text { Swaziland } & 17 & 24 & 70.8(48.9-87.4) \\ \text { Pooled } & 669 & 742 & 88.1(78.1-93.9)\end{array}$

\begin{tabular}{cccccc}
\hline 1 & 1 & 1 & 1 & \\
50 & 60 & 70 & 80 & 90 & 100 \\
& Success versus failure/relapse & $\%$
\end{tabular}
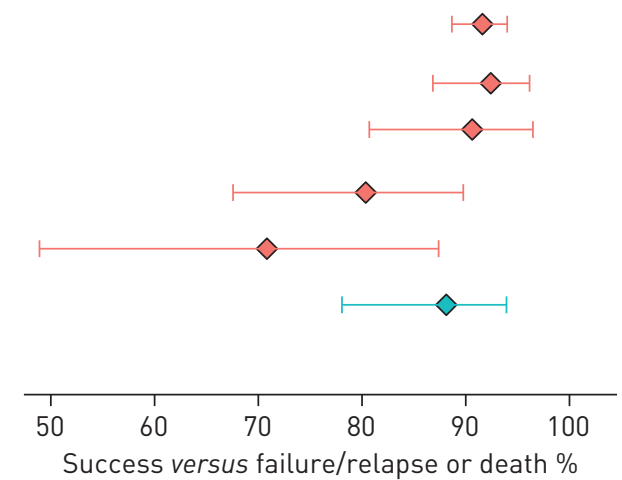

c)

$\begin{array}{lccc}\text { Country } & \text { Success } & \text { Total } & \text { Success \% (95\% CI) } \\ \text { Bangladesh } & 415 & 493 & 84.2(80.7-87.3) \\ \text { Cameroon } & 134 & 150 & 89.3(83.3-93.8) \\ \text { Niger } & 58 & 65 & 89.2(79.1-95.6) \\ \text { Uzbekistan } & 45 & 64 & 70.3(57.6-81.1) \\ \text { Swaziland } & 17 & 24 & 70.8(48.9-87.4) \\ \text { Pooled } & 669 & 796 & 83.0(71.9-90.3)\end{array}$

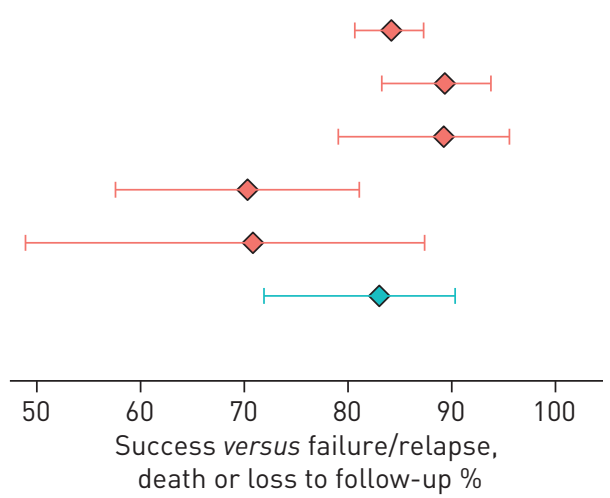

FIGURE 1 Forest plots of treatment outcomes from aggregate data random-effects meta-analyses of five studies: a) success versus failure/relapse, b) success versus failure/relapse or death and c) success versus failure/relapse, death or loss to follow-up. Between-study heterogeneity was significant for success versus failure/relapse. Heterogeneity estimates: success versus failure/relapse 2.1 (95\% $\mathrm{Cl} 0.4-16.2)$, success versus failure/relapse or death $0.2(95 \% \mathrm{Cl} \mathrm{0-1.6)}$ and success versus failure/relapse, death or loss to follow-up 0.2 (95\% Cl 0-1.3).

\section{Adverse events}

The two ongoing studies in Uzbekistan and Swaziland documented adverse events using the DAIDS system. In the Bangladesh study, adverse events recorded corresponded to at least DAIDS grade 2, but were not further stratified. For the other studies in Niger and Cameroon, we were able to assign a DAIDS severity for some types of adverse events using published data and supplemental information from investigators. Grade 3 or 4 adverse events were reported in four studies and occurred in 55 out of 303 (18.1\%, 95\% CI 12.1-26.2\%) participants. Adverse events by system are enumerated in table 7 . Grade 2 or worse hearing loss was reported in five studies $(\mathrm{n}=732)$, with proportions ranging from $0 \%$ (Uzbekistan) to $23 \%$ (Cameroon). Hyperglycaemia was observed in the two studies that used high-dose gatifloxacin (in 
TABLE 4 Results of individual patient data univariable meta-regression to identify risk factors for failure/relapse, death and loss to follow-up

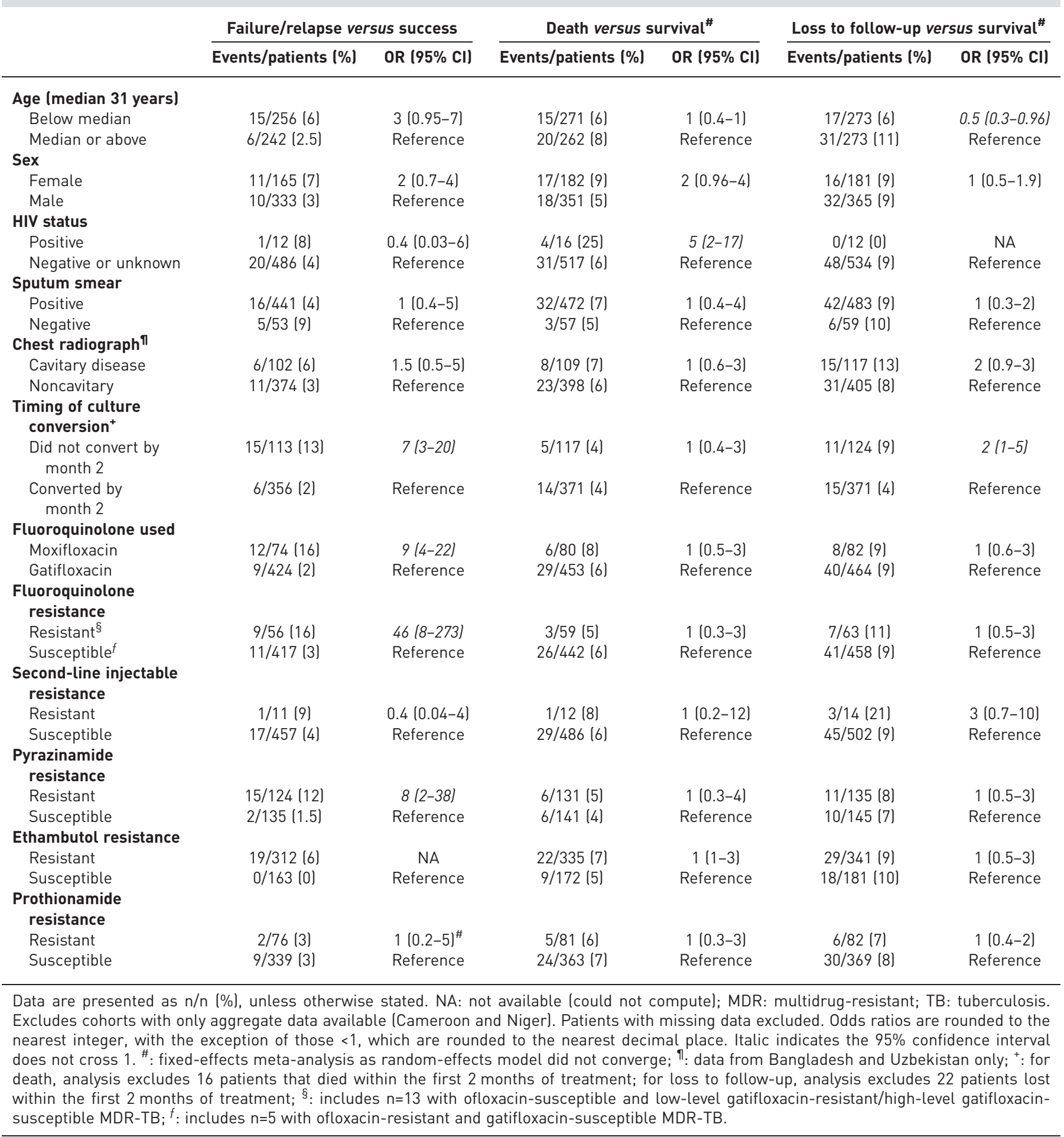

21 out of 493 (4\%) participants in Bangladesh and six out of 65 (9\%) in Niger), but not in the other studies.

\section{Discussion}

In the studies included in our meta-analysis, $29 \%$ of patients with confirmed MDR-TB did not initiate a shorter regimen; these exclusions were mostly due to prior exposure to second-line drugs, baseline drug 
TABLE 5 Treatment outcomes stratified by susceptibility to fluoroquinolones and pyrazinamide, from individual patient data meta-analyses

\begin{tabular}{lcccc} 
Resistance profile & Studies & Success/total & Method of pooling & Success versus fail/relapse \\
\hline FQ-S, PZA-S & 3 & $121 / 121$ & Simple & $100(97-100)$ \\
FQ-S, PZA-R & 3 & $88 / 96$ & $\mathrm{RE}$ & $92(24-100)$ \\
FQ-R, PZA-S & 2 & $12 / 14$ & $\mathrm{FE}$ & $86(54-97)$ \\
FQ-R, PZA-R $^{+}$ & 1 & $19 / 26$ & $\mathrm{NA}$ & $73(52-87)$
\end{tabular}

Data are presented as n, n/n or \% (95\% Cl), unless otherwise stated. Excludes death and loss to follow-up. FQ: fluoroquinolone; PZA: pyrazinamide; R: resistant; S: susceptible; RE: random-effects meta-analysis; FE: fixed-effects meta-analysis; NA: not applicable because only one study; MIC: minimum inhibitory concentration. " : percent calculated as 100xunweighted proportion; " : the FQ-R group is comprised of one participant from the Swaziland cohort with the remaining participants from the Bangladesh cohort; ${ }^{+}$: all data from the Bangladesh cohort. In a study by Aung et al. [7] reporting results from the same Bangladesh cohort, high-level gatifloxacin resistance (defined as MIC $\geqslant 2 \mathrm{mg} \cdot \mathrm{L}^{-1}$ ) was associated with unsuccessful treatment, but not low-level gatifloxacin resistance. In the table, among persons with ofloxacin-resistant multidrug-resistant tuberculosis, high-level gatifloxacin resistance was documented in $n=15$, low-level gatifloxacin resistance in $n=13$ and gatifloxacin MIC was not measured in $n=4$.

resistance, or pre-treatment loss to follow-up or death. Among participants with confirmed MDR-TB that initiated a shorter regimen, $83 \%$ were successfully treated; however, success rates were lower (70\%) in the more recent studies in Uzbekistan and Swaziland. Failure/relapse was more frequent in participants treated with moxifloxacin, with strains resistant to fluoroquinolones or pyrazinamide and in the absence of culture conversion by month 2 of treatment. Based in part on these analyses, the WHO issued a conditional recommendation that shorter regimens may be used in MDR-TB patients not previously treated with second-line drugs, and in whom fluoroquinolone and second-line injectable resistance have been excluded or are "highly unlikely" [8].

The pooled estimates of treatment success rates should be interpreted with caution. In part this is because of the careful selection of participants into the shorter regimen studies that likely resulted in the exclusion of patients at greater risk of failure, death or loss to follow-up. Differences in success rates between our meta-analysis of shorter regimens and a prior meta-analysis of older studies [2], as well as with programmatic outcomes (table 8), are mostly accounted for by deaths and losses to follow-up; this suggests that differences in patient populations (in part due to participant selection), or treatment support, may partially explain the greater success rates in the shorter regimen studies and that similar results may not be achieved in programmatic settings. The heterogeneity demonstrated by the lower success rates in the Uzbekistan and Swaziland cohorts also raises concerns about generalisability. Ultimately, randomised trials comparing shorter regimens to conventional regimens are needed to overcome limitations in the existing evidence base arising from potential selection bias or residual confounding.

Our findings underscore the importance of performing baseline DSTs to fluoroquinolones and pyrazinamide given their association with risk of failure/relapse, and also DSTs for second-line injectables, as the shorter regimen has never been studied in patients with strains that are resistant to the second-line injectable used in their regimen. While some may question the value of baseline DSTs given that the

TABLE 6 Acquired drug resistance

Drug Participants evaluated for acquired resistance
Participants with acquired resistance/evaluated, stratified by treatment outcome

Success ${ }^{\# \quad \text { Failure/relapse Loss to follow-up }}$

$\begin{array}{lcccc}\text { Fluoroquinolones } & 10 & 0 / 3 & 1 / 5 & 0 / 2 \\ \text { Kanamycin } & 18 & 0 / 5 & 2 / 10 & 0 / 3 \\ \text { Ethionamide } & 1 & & 1 / 1 & \\ \text { Pyrazinamide } & 1 & & 1 / 1 & \\ \text { Clofazimine } & 9 & 0 / 2 & 2 / 7\end{array}$

Data are presented as $\mathrm{n}$ or $\mathrm{n} / \mathrm{n}$ from three studies with individual patient data. All acquired resistance was observed in four participants (Bangladesh, $n=2$; Uzbekistan, $n=1$; Swaziland, $n=1$ ). \#: successfully treated participants who had drug susceptibility tests performed on positive cultures obtained during treatment. 


\begin{tabular}{|c|c|c|c|c|c|c|c|c|c|}
\hline & \multicolumn{9}{|c|}{ Severity } \\
\hline & Niger & Cameroon & Uzbekistan & Swaziland & $\frac{\text { Grade } \geqslant 2}{\text { Bangladesh }}$ & Niger & Uzbekistan & Swaziland & $\frac{\text { Not specified }}{\text { Cameroon }}$ \\
\hline Allergic & & & $1(2)$ & & $13(3)$ & & $1(2)$ & & \\
\hline Hyperglycaemia & & & & & $21(4)$ & $6(9)$ & & & \\
\hline Gastrointestinal & & & $1(2)$ & $1(4)$ & $105(21)$ & & $9(14)$ & $3(13)$ & ${ }^{\#}$ \\
\hline Musculoskeletal & & & 2 (3) & & $13(3)$ & & $5(8)$ & & \\
\hline Neurological & & & $3(5)$ & $1(4)$ & $10(2)$ & & $3(5)$ & $1(4)$ & \\
\hline Ophthalmological & $2(3)$ & & & $1(4)$ & $3(1)$ & & & & $1(1)^{\pi}$ \\
\hline Ototoxicity (hearing loss) & $9(14)$ & $24(23)^{\S}$ & & $4(17)$ & $24(5)$ & $4(6)$ & & $2(8)^{+}$ & $22(21)^{\uparrow . \S}$ \\
\hline Psychiatric & & & 2 (3) & $1(4)$ & $1(0)$ & & & & \\
\hline Renal & & $1(1)$ & $2(3)$ & & & & $8(13)$ & $2(8)$ & $1(1)^{\pi}$ \\
\hline
\end{tabular}

Data are presented as $\mathrm{n}(\%)$. Bangladesh, $\mathrm{n}=493$; Cameroon, $\mathrm{n}=150$; Niger, $\mathrm{n}=65$; Uzbekistan, $\mathrm{n}=64$; Swaziland, $\mathrm{n}=24 .{ }^{\#}$ : study reported "many patients" experiencing nausea and vomiting, without specifying number; ${ }^{\text {१: }}$ hepatic: transaminitis necessitating discontinuation of pyrazinamide; ophthalmological: suspected retrobulbar neuritis necessitating discontinuation of ethambutol; ototoxicity: "mild" hearing loss; renal: hypercreatinaemia necessitating dose reduction of kanamycin; ${ }^{+}$: includes one participant with vestibular toxicity; ${ }^{\S}$ : denominator for ototoxicity is 106 participants who had two audiograms performed (at baseline and 4 months).

majority of participants with strains resistant to fluoroquinolones or pyrazinamide were successfully treated $(>80 \%)$, as were the majority with strains resistant to both drugs $(73 \%)$, the crude rates should be interpreted with caution due to the small numbers of participants with resistance and the careful participant selection. Unfortunately, data were insufficient to determine the importance of baseline resistance to other component medications.

The present review has a number of limitations. First, the preponderance of patient data originating from the Bangladesh study. However, in a sensitivity analysis where this study was excluded, the pooled proportion of treatment success versus failure/relapse death or default was similar to that reported in our primary analysis $(82.4 \%, 95 \%$ CI $63.0-92.8 \%)$. Second, the exclusion of one ongoing, unpublished study. However, this is unlikely to have affected our findings or conclusions because the excluded study had interim participant selection and outcome rates (publicly available in the WHO guidelines [13]) that were similar to the studies that we included [8]. Third, all meta-regression analyses were unadjusted for potential confounders, due to limited number of outcomes.

TABLE 8 Comparison of pooled multidrug-resistant tuberculosis (MDR-TB) treatment outcomes from a meta-analysis of studies of short regimens to pooled outcomes from a meta-analysis of older MDR-TB studies and to outcomes pooled from programmatic data reported by the World Health Organization (WHO)

\begin{tabular}{lccc} 
& $\begin{array}{c}\text { Meta-analysis of short } \\
\text { regimens }\end{array}$ & $\begin{array}{c}\text { Meta-analysis of older } \\
\text { studies }\end{array}$ & $\begin{array}{c}\text { Programmatic } \\
\text { data }\end{array}$ \\
\hline Subjects n & 796 & 9153 & 86936 \\
Treatment success \% & 83 & 54 & 52 \\
Failure/relapse \% & 3 & 8 & 9 \\
Death \% & 6 & 15 & 17 \\
Loss to follow-up or no & 5 & 23 & 22 \\
$\quad$ outcome data \% & & &
\end{tabular}

\#: the meta-analysis of older MDR-TB studies [2] pooled outcomes from cohorts whose participants and treatment differed in many aspects, e.g. history of prior exposure to second-line drugs, baseline drug resistance, use of individualised versus standardised regimens, number and selection of drugs, and the duration of intensive and continuation phases (this older meta-analysis also included 603 patients treated with the short regimen in the Bangladesh cohort, some of whom were included in the present meta-analysis of shorter regimens); " : MDR-TB cohorts started on treatment in 2013, reported to the WHO as part of aggregated reporting by countries worldwide [1]. 
Our study also has several strengths. First, the use of IPD meta-analysis that enabled us to identify patient-level risk factors for failure/relapse, death and loss to follow-up, which would not have been possible with a traditional, aggregate data meta-analysis. Second, the inclusion of unpublished data from two studies in Uzbekistan and Swaziland, which allowed us to identify that the high success rates reported in the published studies may not be generalisable. Third, the quality of the data, which included at least 1-year of follow-up for relapse in all studies.

Our study has implications for the programmatic use of shorter MDR-TB regimens. First, our observations that resistance to fluoroquinolones or resistance to pyrazinamide were risk factors for failure/relapse and also that acquired drug resistance occurred among failures underscore that programmes implementing shorter regimens should simultaneously strive to scale-up DSTs for the component drugs, as others have recently suggested [14-16], and have alternative regimens available for patients identified to have strains that are resistant at baseline or that acquire resistance during treatment. This is also supported by evidence from other studies showing that successful MDR-TB treatment is determined, in part, by the number of drugs used against which the infecting strain is susceptible [2, 17-19], including pyrazinamide [20]. Second, all shorter regimen studies used daily directly observed therapy with social support and provided antiretroviral therapy to all people living with HIV; hence, such interventions should be considered essential components of shorter regimens. Third, programmes should ensure proper screening and management of adverse events [21]. Fourth, successfully treated patients should be followed for relapse for at least 1 year after completion of therapy.

\section{Conclusions}

The high rate of treatment success that we estimated from observational studies of shorter standardised regimens is promising, but its generalisability to less selected populations and to programmatic settings is uncertain. Our data suggest that key determinants of treatment success with these regimens include baseline susceptibility to fluoroquinolones, pyrazinamide and second-line injectables. The generalisability of the evidence base will be strengthened by more data on baseline resistance to other component drugs, on the regimen's effectiveness in programmatic settings and from randomised trials comparing shorter standardised regimens to those of more conventional duration.

\section{Acknowledgements}

We thank Armand Van Deun (Institute of Tropical Medicine, Antwerp, Belgium), Aung Kya Jai Maug (Damien Foundation, Dhaka, Bangladesh), Nargiza Parpieva (Ministry of Health, Tashkent, Uzbekistan), Mirzagaleb N. Tillyashaykhov (Ministry of Health, Tashkent, Uzbekistan), Jürgen Noeske (Independent Consultant, NTP, Yaounde, Cameroon), Alberto Piubello (International Union Against Tuberculosis and Lung Disease (the Union) and Damien Foundation, Niamey, Niger), Valérie Schwoebel (the Union, Paris, France) and Arnaud Trébucq (the Union, Paris, France). We thank the country and headquarters staff of the Damien Foundation (Dhaka, Bangladesh), Uzbekistan Ministry of Health (Nukus, Uzbekistan), Médecins Sans Frontières (MSF) Uzbekistan (Nukus, Uzbekistan), National TB Control Programme Swaziland (Manzini, Swaziland), MSF Swaziland (Manzini, Swaziland) and MSF Operational Center Amsterdam (London, UK and Amsterdam, The Netherlands).

\section{References}

1 WHO. Global Tuberculosis Report. Geneva, World Health Organization, 2016

2 Ahuja SD, Ashkin D, Avendano M, et al. Multidrug resistant pulmonary tuberculosis treatment regimens and patient outcomes: an individual patient data meta-analysis of 9,153 patients. PLoS Med 2012; 9: e1001300.

3 WHO. Guidelines for the Programmatic Management of Drug-Resistant Tuberculosis - 2011 update. Geneva, World Health Organization, 2011.

4 Nathanson E, Gupta R, Huamani P, et al. Adverse events in the treatment of multidrug-resistant tuberculosis: results from the DOTS-Plus initiative. Int J Tuberc Lung Dis 2004; 8: 1382-1384.

5 Bloss E, Kuksa L, Holtz TH, et al. Adverse events related to multidrug-resistant tuberculosis treatment, Latvia 2000-2004. Int J Tuberc Lung Dis 2010; 14: 275-281.

6 Van Deun A, Maug AK, Hamid Salim MA, et al. Short, highly effective, and inexpensive standardized treatment of multidrug-resistant tuberculosis. Am J Respir Crit Care Med 2010; 182: 684-692.

7 Aung KJ, Van Deun A, Declercq E, et al. Successful '9-month Bangladesh regimen' for multidrug-resistant tuberculosis among over 500 consecutive patients. Int J Tuberc Lung Dis 2014; 18: 1180-1187.

8 WHO. WHO Treatment Guidelines for Drug-Resistant Tuberculosis - 2016 update. Geneva, World Health Organization, 2016.

9 Bastos M, Lan Z, Menzies D. An updated systematic review and meta-analysis for treatment of multidrug-resistant tuberculosis. Eur Respir J 2017; 49: 1600803.

10 Hamza TH, van Houwelingen HC, Stijnen T. The binomial distribution of meta-analysis was preferred to model within-study variability. J Clin Epidemiol 2008; 61: 41-51.

11 Kuaban C, Noeske J, Rieder HL, et al. High effectiveness of a 12-month regimen for MDR-TB patients in Cameroon. Int J Tuberc Lung Dis 2015; 19: 517-524.

12 Piubello A, Harouna SH, Souleymane MB, et al. High cure rate with standardised short-course multidrug-resistant tuberculosis treatment in Niger: no relapses. Int J Tuberc Lung Dis 2014; 18: 1188-1194. 
13 WHO. WHO Treatment Guidelines for Drug-Resistant Tuberculosis - 2016 update. Online appendix. http://apps. who.int/iris/bitstream/10665/250125/5/9789241549639-webannexes-eng.pdf?ua=12016 Date last accessed: March 22, 2017.

14 Sotgiu G, Tiberi S, D'Ambrosio L, et al. Faster for less: the new "shorter" regimen for multidrug-resistant tuberculosis. Eur Respir J 2016; 48: 1503-1507.

15 Sotgiu G, Tiberi S, D'Ambrosio L, et al. WHO recommendations on shorter treatment of multidrug-resistant tuberculosis. Lancet 2016; 387: 2486-2487.

16 Varaine F, Guglielmetti L, Huerga $\mathrm{H}$, et al. Eligibility for the shorter multidrug-resistant tuberculosis regimen: ambiguities in the World Health Organization recommendations. Am J Respir Crit Care Med 2016; 194: $1028-1029$.

17 Cegielski JP, Kurbatova E, van der Walt M, et al. Multidrug-resistant tuberculosis treatment outcomes in relation to treatment and initial versus acquired second-line drug resistance. Clin Infect Dis 2016; 62: 418-430.

18 Falzon D, Gandhi N, Migliori GB, et al. Resistance to fluoroquinolones and second-line injectable drugs: impact on multidrug-resistant TB outcomes. Eur Respir J 2013; 42: 156-168.

19 Migliori GB, Sotgiu G, Gandhi NR, et al. Drug resistance beyond extensively drug-resistant tuberculosis: individual patient data meta-analysis. Eur Respir J 2013; 42: 169-179.

20 Franke MF, Becerra MC, Tierney DB, et al. Counting pyrazinamide in regimens for multidrug-resistant tuberculosis. Ann Am Thorac Soc 2015; 12: 674-679.

21 WHO. Active Tuberculosis Drug-Safety Monitoring and Management (aDSM). Framework for Implementation. Geneva, World Health Organization, 2015. 